\title{
On the Capacity of Fading Channels with Amplitude-Limited Inputs
}

\author{
Ahmad ElMoslimany*and Tolga M. Duman ${ }^{\dagger}$ \\ *Arizona State University, School of Electrical, Computer and Energy Engineering, Tempe, AZ 85287-5706 \\ ${ }^{\dagger}$ Bilkent University, Dept. of Electrical and Electronics Engineering, TR-06800, Bilkent, Ankara, Turkey \\ Emails: aelmosli@asu.edu,duman@ee.bilkent.edu.tr
}

\begin{abstract}
We address the problem of finding the capacity of fading channels under the assumption of amplitude-limited inputs. Specifically, we show that if the fading coefficients have a finite support and the channel state information is only available at the receiver side, there is a unique input distribution that achieves the channel capacity and this input distribution is discrete with a finite number of mass points.
\end{abstract}

\section{INTRODUCTION}

Capacity of channels with amplitude-limited inputs has been studied for the first time by Smith in [1]. In his work, Smith shows that the capacity of scalar Gaussian channels is achieved by a unique input distribution and this distribution is discrete with finite number of mass points. The capacity of fading channels with amplitude-limited inputs has also been studied previously in the literature for certain fading distributions with different input constraints. For instance, the capacity of Rayleigh fading channels where neither the transmitter nor the receiver has the channel state information with an average power constrained input is achieved by a discrete distribution [2]. Capacity of Rician fading channels with inputs having constraints on the second and the fourth moments is achieved by a discrete input distribution as well [3]. In another closely related work [4], the authors generalize the previous results, and show that for any conditionally Gaussian channel with amplitude-limited inputs, the channel capacity is achieved by discrete input distributions.

In this paper, we consider the problem of finding the capacity of fading channels with amplitude-limited inputs where the fading distribution is arbitrary and the channel inputs are amplitude-limited. We assume that the channel gains are real, have finite support and are known at the receiver. We show that the capacity achieving distribution is discrete with a finite number of mass points. We note that the channel model under consideration does not fall within the framework of conditionally Gaussian channels studied in [4], hence its results do not apply. In order to prove our results, we borrow ideas and approach from $[1,5,13]$. Specifically, we show that the capacity optimization problem is convex, then we invoke the Karush-Kuhn-Tucker (KKT) Theorem to derive the optimality conditions. The discreteness of the capacityachieving distribution is shown by adopting techniques from

This work is funded by National Science Foundation under the contract NSF-ECCS 1102357 and by the EC Marie Curie Career Integration Grant PCIG12-GA-2102-334213. complex analysis as in [13] and [5] for scalar Gaussian channels and multiple access channels, respectively.

The paper is organized as follows. In Section II, we present the fading channel model under consideration. In Section III, we provide the required definitions. In Section IV, we show that the capacity is maximized by a unique input distribution. Then, we show, in Section V, that this input distribution is discrete, and we conclude the paper in Section VI with a brief summary.

\section{Channel Model}

We consider a fading channel model where the received signal $Y$ is given by

$$
Y=\alpha X+N
$$

where $X$ is the channel input that is amplitude-constrained to $[-A, A]$, i.e., it has a probability distribution function $F_{X}(x)$ that belongs to the class of probability distribution functions $\mathcal{F}_{X}$ such that for any $F_{X} \in \mathcal{F}_{X}, F_{X}(x)=0$ for any $x<-A$ and $F_{X}(x)=1$ for any $x \geq A$. The coefficient $\alpha$ is the fading channel gain with a probability distribution function $F_{\alpha}(u)$. We assume that $\alpha$ has a finite support, i.e., $\alpha \in\left[0, u_{0}\right]$ for some $u_{0}<\infty$, and that the channel state information is available only at the receiver side. The noise $N$ is Gaussian, i.e., $N \sim \mathcal{N}\left(0, \sigma^{2}\right)$, and it is independent for different uses of the channel. We assume that the input $X$ and the fading coefficient $\alpha$ are also independent.

We emphasize that this model differs from the previous models studied in the literature. The most closely related one is in [4] where the authors study the conditionally Gaussian channels. When the fading gain is zero mean complex Gaussian (i.e., for Rayleigh fading), the channel becomes conditionally Gaussian, and the results of [4] apply. However, here we consider fading channels with an arbitrary (but finite support) distribution, hence our model does not fall within the framework of [4].

The probability density function of the output is given by

$$
f_{Y}\left(y ; F_{X}\right)=\int_{0}^{u_{0}} \int_{-A}^{A} P_{N}(y-u x) d F_{X}(x) d F_{\alpha}(u),
$$

where $P_{N}(y-u x)=f_{Y \mid X, \alpha}(y \mid x, u)$ is the probability density function of the channel output $Y$ conditioned on specific values of $X$ and $\alpha$, and $f_{Y}\left(y ; F_{X}\right)$ is the probability density function of the channel output $Y$ when the input has a prob- 
ability distribution function $F_{X}$. The existence of $f_{Y}\left(y ; F_{X}\right)$ is guaranteed by the existence of $P_{N}$ [6].

In the following, we derive bounds on the probability density function of the noise term $P_{N}(y-u x)$ and the conditional probability density function $f_{Y \mid \alpha}(y \mid u)$ for later use. It is straightforward to show that, for $u>0$, the probability density function is bounded as follows

$$
q(y, u) \leq P_{N}(y-u x) \leq Q(y, u),
$$

where

$$
q(y, u)= \begin{cases}k_{1} \exp \left(-k_{2}(y-u A)^{2}\right) & \text { if } y \leq 0 \\ k_{1} \exp \left(-k_{2}(y+u A)^{2}\right) & \text { if } y>0\end{cases}
$$

and

$$
Q(y, u)= \begin{cases}k_{3} \exp \left(-k_{4}(y+u A)^{2}\right) & \text { if } y<-u A \\ k_{3} & \text { if } y \in[-u A, u A] \\ k_{3} \exp \left(-k_{4}(y-u A)^{2}\right) & \text { if } y>u A\end{cases}
$$

for some finite and positive $k_{1}, k_{2}, k_{3}$, and $k_{4}$. As a result, the conditional probability density function $f_{Y \mid \alpha}(y \mid u)$ can be bounded as well

$$
\gamma(y, u) \leq f_{Y \mid \alpha}(y \mid u) \leq \Gamma(y, u)
$$

where

$$
\gamma(y, u)=q(y, u), \text { and } \Gamma(y, u)=Q(y, u) .
$$

\section{DEFINITIONS AND PRELIMINARIES}

The average mutual information between the input and the output conditioned on the channel gain is defined as $[7,8]$

$$
I_{F_{X}}(X ; Y \mid \alpha) \triangleq \int_{0}^{u_{0}} I_{F_{X}}(X ; Y \mid \alpha=u) d F_{\alpha}(u)
$$

where

$I_{F_{X}}(X ; Y \mid \alpha=u) \triangleq \int_{-\infty}^{\infty} \int_{-A}^{A} P_{N}(y-u x) \log \left(\frac{P_{N}(y-u x)}{f_{Y \mid \alpha}\left(y \mid u ; F_{X}\right)}\right) d F_{X}(x) d y$

and $f_{Y \mid \alpha}\left(y \mid u ; F_{X}\right)$ is the conditional probability density function of the output which is defined as

$$
f_{Y \mid \alpha}\left(y \mid u ; F_{X}\right)=\int_{-A}^{A} P_{N}(y-u x) d F_{X}(x) .
$$

We define the conditional entropy $H_{F_{X}}(Y \mid \alpha)$ as

$$
H_{F_{X}}(Y \mid \alpha)=\int_{0}^{u_{0}} H_{F_{X}}(Y \mid \alpha=u) d F_{\alpha}(u) \text {. }
$$

where

$$
H_{F_{X}}(Y \mid \alpha=u) \triangleq-\int_{-\infty}^{\infty} f_{Y \mid \alpha}\left(y \mid u ; F_{X}\right) \log f_{Y \mid \alpha}\left(y \mid u ; F_{X}\right) d y .
$$

For noise with finite variance and bounded density function, the conditional mutual information function can be written as

$$
I_{F_{X}}(X ; Y \mid \alpha)=H_{F_{X}}(Y \mid \alpha)-D,
$$

where $D$ is the noise entropy

$$
D \triangleq-\int_{-\infty}^{\infty} P_{N}(z) \log P_{N}(z) d z .
$$

For Gaussian noise with mean 0 and variance $\sigma^{2}$, the entropy is

$$
D=\frac{1}{2} \log \left(2 \pi e \sigma^{2}\right) .
$$

The channel capacity is defined as

$$
C=\max _{F_{X} \in \mathcal{F}_{X}} I_{F_{X}}(X ; Y \mid \alpha) \text {. }
$$

We define the conditional mutual information density $i_{F}(x \mid \alpha=u)$ and the conditional entropy density $h_{F}(x \mid \alpha=u)$ both conditioned on a specific value of $\alpha$ as

$$
\begin{aligned}
& i_{F_{X}}(x \mid \alpha=u) \triangleq \int_{-\infty}^{\infty} P_{N}(y-u x) \log \frac{P_{N}(y-u x)}{f_{Y \mid \alpha}\left(y \mid u ; F_{X}\right)} d y, \\
& h_{F_{X}}(x \mid \alpha=u) \triangleq-\int_{-\infty}^{\infty} P_{N}(y-u x) \log f_{Y \mid \alpha}\left(y \mid u ; F_{X}\right) d y .
\end{aligned}
$$

Thus, the following equation holds

$$
i_{F_{X}}(x \mid \alpha=u)=h_{F_{X}}(x \mid \alpha=u)-D .
$$

Define the conditional mutual information density $i_{F_{X}}(x \mid \alpha)$ and the conditional entropy density as $h_{F_{X}}(x \mid \alpha)$

$$
\begin{gathered}
i_{F_{X}}(x \mid \alpha) \triangleq \int_{0}^{u_{0}} i_{F_{X}}(x \mid \alpha=u) d F_{\alpha}(u), \\
h_{F_{X}}(x \mid \alpha) \triangleq \int_{0}^{u_{0}} h_{F_{X}}(x \mid \alpha=u) d F_{\alpha}(u) .
\end{gathered}
$$

Thus, we can write

$$
\begin{gathered}
i_{F_{X}}(x \mid \alpha)=h_{F_{X}}(x \mid \alpha)-D, \\
I_{F_{X}}(X ; Y \mid \alpha)=\int_{-A}^{A} i_{F_{X}}(x \mid \alpha) d F_{X}(x), \\
H_{F_{X}}(Y \mid \alpha)=\int_{-A}^{A} h_{F_{X}}(x \mid \alpha) d F_{X}(x) .
\end{gathered}
$$

These equations hold by the definition of the information density and the definition of the entropy density. We note that in the previous expressions the order of integrals has been changed which can be justified using Fubini's theorem by showing that the mutual information density and the entropy density are finite as shown in Lemma 1.

Lemma 1. The conditional entropy $H_{F_{X}}(Y \mid \alpha)$ and the conditional mutual information $I_{F_{X}}(X ; Y \mid \alpha)$ are finite.

Proof: It is sufficient to show the finiteness of $H_{F_{X}}(Y \mid \alpha)$ as the difference between $I_{F_{X}}(X ; Y \mid \alpha)$ and $H_{F_{X}}(Y \mid \alpha)$ is just a constant. We can write

$$
\begin{aligned}
& \left|H_{F_{X}}(Y \mid \alpha)\right| \\
& =\left|\int_{0}^{u_{0}} H_{F_{X}}(Y \mid \alpha=u) d F_{\alpha}(u)\right| \\
& \leq \int_{-A}^{A} \int_{0}^{u_{0}} \int_{-\infty}^{\infty}\left|P_{N}(y-u x) \log \left(f_{Y \mid \alpha}\left(y \mid u ; F_{X}\right)\right)\right| d y d F_{\alpha}(u) d F_{X}(x) \\
& \leq \int_{-A}^{A} \int_{0}^{u_{0}} \int_{-\infty}^{\infty} P_{N}(y-u x)\left[-\log \left(f_{Y \mid \alpha}\left(y \mid u ; F_{X}\right)\right)\right. \\
& \left.\quad+2\left|\log \left(k_{3}\right)\right|\right] d y d F_{\alpha}(u) d F_{X}(x) \\
& \leq \int_{-A}^{A} \int_{0}^{u_{0}} \int_{-\infty}^{\infty} Q(y, u)\left[-\log (q(y, u))+2\left|\log \left(k_{3}\right)\right|\right] d y d F_{\alpha}(u) d F_{X}(x) .
\end{aligned}
$$

The right hand side can be easily shown to be finite. Hence we can conclude that $H_{F_{X}}(Y \mid \alpha)$ and $I_{F_{X}}(X ; Y \mid \alpha)$ are both finite. 


\section{CAPACity Optimization PROBlem}

In this section, we show that the mutual information is a strictly concave, weakly differentiable, and continuous function of the input distribution.

\section{A. The Mutual Information is a Continuous Function of the} Distribution

The conditional mutual information is

$$
I_{F_{X}}(X ; Y \mid \alpha)=H_{F_{X}}(Y \mid \alpha)-D,
$$

and the conditional entropy is

$$
H_{F_{X}}(Y \mid \alpha)=\int_{0}^{u_{0}} H_{F_{X}}(Y \mid \alpha=u) d F_{\alpha}(u) .
$$

In order to show the continuity of $H_{F_{X}}(Y \mid \alpha)$, we show that for any sequence of the input distribution functions, $H_{F_{X}^{(n)}}(Y \mid \alpha=$ $u)$ is bounded by an integrable function and hence we can invoke the Dominated Convergence Theorem. That is, let us fix a sequence $\left\{F_{X}^{(n)}(x)\right\}_{n \geq 1}$ in $\mathcal{F}_{X}$ such that $F_{X}^{(n)}(x) \rightarrow F_{X}(x)$ for some $F_{X} \in \mathcal{F}_{X}$. From (6),

$$
\begin{aligned}
& \left|-f_{Y \mid \alpha}\left(y \mid u ; F_{X}^{(n)}\right) \log \left(f_{Y \mid \alpha}\left(y \mid u ; F_{X}^{(n)}\right)\right)\right| \\
& \quad \leq \Gamma(y, u)\left[-\log (\gamma(y, u))+2\left|\log \left(k_{3}\right)\right|\right] .
\end{aligned}
$$

As a result

$$
\begin{aligned}
& \left|H_{F_{X}^{(n)}}(Y \mid \alpha=u)\right| \\
& \quad=\left|\int_{-\infty}^{\infty}-f_{Y \mid \alpha}\left(y \mid u ; F_{X}^{(n)}\right) \log \left(f_{Y \mid \alpha}\left(y \mid u ; F_{X}^{(n)}\right)\right) d y\right|, \\
& \quad \leq \int_{-\infty}^{\infty}\left|-f_{Y \mid \alpha}\left(y \mid u ; F_{X}^{(n)}\right) \log \left(f_{Y \mid \alpha}\left(y \mid u ; F_{X}^{(n)}\right)\right)\right| d y, \\
& \quad \leq \int_{-\infty}^{\infty} \Gamma(y, u)\left[-\log (\gamma(y, u))+2\left|\log \left(k_{3}\right)\right|\right] d y<\infty .
\end{aligned}
$$

It is easy to verify that $\left|\int_{0}^{u_{0}} H_{F_{X}^{(n)}}(Y \mid \alpha=u) d F_{\alpha}(u)\right|<\infty$. Thus, we can invoke the Dominated Convergence Theorem to show that

$$
\begin{aligned}
\lim _{n \rightarrow \infty} H_{F_{X}^{(n)}}(Y \mid \alpha) & =\lim _{n \rightarrow \infty} \int_{0}^{u_{0}} H_{F_{X}^{(n)}}(Y \mid \alpha=u) d F_{\alpha}(u), \\
& =\int_{0}^{u_{0}} \lim _{n \rightarrow \infty} H_{F_{X}^{(n)}}(Y \mid \alpha=u) d F_{\alpha}(u), \\
& =H_{F_{X}}(Y \mid \alpha) .
\end{aligned}
$$

Hence the conditional entropy is a continuous function of the input distribution. Since the difference between the conditional entropy and the conditional mutual information is just a constant, we conclude that the conditional mutual information is also continuous.

\section{B. The Mutual Information is a Strictly Concave Function of the Input Distribution}

We have

$$
I_{F_{X}}(Y ; X \mid \alpha)=H_{F_{X}}(Y \mid \alpha)-D \text {. }
$$

Hence, it is enough to show that the conditional entropy $H_{F_{X}}(Y \mid \alpha)$ is a strictly concave function of the distribution to conclude the strict concavity of the mutual information function. The conditional entropy is given by

$$
H_{F_{X}}(Y \mid \alpha)=\int_{0}^{u_{0}} H_{F_{X}}(Y \mid \alpha=u) d F_{\alpha}(u) \text {. }
$$

To show the strict concavity of the conditional entropy, we first show that $H_{F_{X}}(Y \mid \alpha=u)$ is strictly concave for every $u$ in the support of the random variable $\alpha$ by considering

$$
Y=u X+N \text {. }
$$

for a fixed $u$. For $u>0$, we define a new random variable $Y^{\prime}=\frac{Y}{u}$, i.e.,

$$
Y^{\prime}=X+\frac{N}{u}
$$

We assume that $F_{\alpha}(0) \neq 1$, i.e., the measure of the set of the nonzero values of the channel coefficients is not zero. Thus, the equivalent model in (31) is the same as the scalar Gaussian channel model studied by Smith in [9] which leads to the strict concavity of the conditional entropy for a given $u$, i.e., $H_{F_{X}}(Y \mid \alpha=u)$ is a strictly concave function. As a result, we conclude the strict concavity of the conditional output entropy since positive weighted sum of strictly concave functions is strictly concave [10].

\section{The Mutual Information is a Weakly Differentiable Func- tion}

Lemma 2. The mutual information function $I(X ; Y \mid \alpha)$ is a weakly differentiable function and its weak derivative is

$$
I_{F_{1}, F_{2}}^{\prime}(X ; Y \mid \alpha)=\int_{-A}^{A} i_{F_{1}}(x \mid \alpha) d F_{2}(x)-I_{F_{1}}(X ; Y \mid \alpha) \text {. }
$$

Proof: The proof follows similar line of arguments as in $[1,5]$. The details are provided in [6].

We note that similar results up to this point has been reported before in [11] (which considers a more general setup), however, for the sake of completeness and in order to establish notation, and also to make the paper self contained, we have included the required definitions and proofs.

Theorem 1. $C$, the capacity of the channel, is achieved by a unique probability distribution function $F_{0}$ in $\mathcal{F}_{X}$, i.e.,

$$
C \triangleq \max _{F_{X} i n \mathcal{F}_{X}} I(X ; Y \mid \alpha) \text {. }
$$

Furthermore, the necessary and sufficient conditions on the optimal input distribution are

$$
\begin{aligned}
& i_{F_{0}}(x \mid \alpha) \leq I_{F_{0}}(X ; Y \mid \alpha), \quad \forall x \in[-A, A], \\
& i_{F_{0}}(x \mid \alpha)=I_{F_{0}}(X ; Y \mid \alpha), \quad \forall x \in E_{0} .
\end{aligned}
$$

Proof: The space $\mathcal{F}_{X}$ is convex and compact in some topology [1]. Earlier in this section we showed that the function $I: \mathcal{F}_{X} \rightarrow \mathbb{R}$ is strictly concave, continuous, and weakly differentiable in $\mathcal{F}_{X}$. By invoking the KKT conditions and following the standard arguments as in Smith [1], the necessary and sufficient conditions can be derived. The details are provided in [6].

\section{Discreteness of the Optimal Distribution}

In this subsection, we prove that the optimal distribution that maximizes the mutual information function is discrete 
with a finite number of mass points. In a nutshell, we show that the extension of the conditional entropy to the complex plane is well defined and this extension is analytic. Then, we assume that the set of points of increase of the input probability distribution function $E_{0}$ contains an infinite number of elements. Finally, Bolzano-Weierstrass and Identity Theorems are invoked to show that the assumption of the non-finiteness of $E_{0}$ leads to a contradiction.

The conditional entropy density is given by

$$
h_{F_{0}}(x \mid \alpha)=\int_{0}^{u_{0}} h_{F_{0}}(x \mid \alpha=u) d F_{\alpha}(u) .
$$

We first extend $h_{F_{0}}(x \mid \alpha=u)$ to the complex plane. For any $z=\eta+i \zeta \in \mathbb{C}$ and $u \in\left[0, u_{0}\right]$

$$
\begin{aligned}
& |h(z \mid \alpha=u)| \\
& \quad \leq \int_{-\infty}^{\infty}\left|P_{N}(y-u z)\right|\left|\log f_{Y \mid \alpha}\left(y \mid u ; F_{X}\right)\right| d y, \\
& \quad=\int_{-\infty}^{\infty} \frac{1}{\sqrt{2 \pi \sigma^{2}}}\left|\exp \left(\frac{-(y-u z)^{2}}{2 \pi \sigma^{2}}\right)\right| \\
& \quad \leq \quad \int_{-\infty}^{\infty} \frac{1}{\sqrt{2 \pi \sigma^{2}}}\left|\exp \left(\frac{-(y-u \eta-i u \zeta)^{2}}{2 \pi \sigma^{2}}\right)\right| \\
& \leq \frac{1}{\sqrt{2 \pi \sigma^{2}}} \exp \left(\frac{u \zeta^{2}}{2 \pi \sigma^{2}}\right) \int_{-\infty}^{\infty}\left|\exp \left(\frac{-(y-u \eta)^{2}}{2 \pi \sigma^{2}}\right)\right| \\
& \quad\left[-\log \left(k_{1}\right)+k_{2}\left|(y-u z)^{2}\right|+2\left|\log \left(k_{3}\right)\right|\right] d y, \\
& \leq \exp \left(\frac{u \zeta^{2}}{2 \pi \sigma^{2}}\right) \int_{-\infty}^{\infty}\left|P_{N}(y-u \eta)\right| \\
& \quad\left[-\log \left(k_{1}\right)+k_{2}\left|(y-u z)^{2}\right|+2\left|\log \left(k_{3}\right)\right|\right] d y<\infty .
\end{aligned}
$$

Hence $|h(z \mid \alpha=u)|$ is finite for any $|z|<\infty$. Thus, the extension of $h_{F_{X}}(z \mid \alpha=u)$ is well defined.

Since $\exists B<\infty$, such that $\forall u \in\left[0, u_{0}\right]$ we have $\left|h_{F_{X}}(z \mid \alpha=u)\right| \leq B$, i.e.,

$$
\begin{aligned}
\left|h_{F_{0}}(z \mid \alpha)\right| & =\left|\int_{0}^{u_{0}} h_{F_{X}}(z \mid \alpha=u) d F_{\alpha}(u)\right|, \\
& \leq \int_{0}^{u_{0}}\left|h_{F_{X}}(z \mid \alpha=u)\right| d F_{\alpha}(u), \\
& \leq B \int_{0}^{u_{0}} d F_{\alpha}(u)=B<\infty,
\end{aligned}
$$

hence $h_{F_{0}}(z \mid \alpha)$ has an extension to the complex plane as well.

Since $P_{N}(\cdot)$ is an analytic function, using the Cauchy Integral Theorem [12] we have

$$
\oint_{\omega} P_{N}(z) d z=0
$$

where $\omega$ is any simple closed contour on the complex plane. To show the analyticity of the conditional entropy density, we use Morera's Theorem, i.e., by showing that the integration of the conditional entropy over any simple closed contour is zero, we can conclude that the function is analytic. That is,

$$
\begin{aligned}
& \oint_{\omega} h_{F_{X}}(z \mid \alpha) d z \\
& \quad=\oint_{\omega} \int_{0}^{u_{0}} \int_{-\infty}^{\infty}-P_{N}(y-u z) \log \left(f_{Y \mid \alpha}\left(y \mid u ; F_{X}\right)\right) d F_{\alpha}(u) d y d z \\
& \quad \stackrel{(a)}{=} \int_{0}^{u_{0}} \int_{-\infty}^{\infty}-\log \left(f_{Y \mid \alpha}\left(y \mid u ; F_{X}\right)\right) \oint_{\omega} P_{N}(y-u z) d z d F_{\alpha}(u) d y=0,
\end{aligned}
$$

where the order of integrals in $(a)$ is changed by invoking the Fubini's Theorem that requires the finiteness of $\oint_{\omega}\left|h_{F_{X}}(z \mid \alpha)\right| d z$. This can be justified as follows: we define $M_{\omega}$ as

$$
M_{\omega}=\max _{z \in \omega}\left|h_{F_{X}}(z \mid \alpha)\right| .
$$

$M_{\omega}$ exists since the conditional entropy $\left|h_{F_{X}}(z \mid \alpha)\right|$ is bounded, continuous in $z$, and the contour $\omega$ is closed. Hence,

$$
\begin{aligned}
& \left|\oint_{\omega} h_{F_{X}}(z \mid \alpha) d z\right| \\
& =\left|\oint_{\omega} \int_{0}^{u_{0}} \int_{-\infty}^{\infty}-P_{N}(y-u z) \log \left(f_{Y \mid \alpha}\left(y \mid u ; F_{X}\right)\right) d F_{\alpha}(u) d y d z\right|, \\
& \leq \oint_{\omega}\left|\int_{-\infty}^{\infty} \int_{0}^{u_{0}}-P_{N}(y-u z) \log \left(f_{Y \mid \alpha}\left(y \mid u ; F_{X}\right)\right) d F_{\alpha}(u) d y\right| d z, \\
& \leq \oint_{\omega} M_{\omega} d z, \\
& \leq M_{\omega} l_{\omega}<\infty,
\end{aligned}
$$

where $l_{\omega}$ is the length of $\omega$ which is finite as $\omega$ is a closed contour.

Therefore, we establish that the extension of the conditional mutual information density $i_{F_{0}}(z \mid \alpha)$ to the complex plane is well defined (since its difference with the entropy density is a constant), and it is analytic.

We prove the discreteness of the capacity-achieving distribution by a contradiction. We first assume that the set of points of increase $E_{0}$ has an infinite cardinality. From the optimality condition in (35) we have

$$
\int_{0^{+}}^{u_{0}}\left(i_{F_{0}}(x \mid \alpha=u)-I_{F_{0}}(X ; Y \mid \alpha=u)\right) d F_{\alpha}(u)=0,
$$

$\forall x \in E_{0}$. Since $E_{0}$ is bounded, it has a limit point (BolzanoWeierstrass Theorem). The conditional mutual information density $i_{F_{0}}(z \mid \alpha)$ is analytic on the entire complex plane. That is, we can invoke the Identity Theorem to show that the optimality condition is

$$
\int_{0^{+}}^{u_{0}}\left(i_{F_{0}}(x \mid \alpha=u)-I_{F_{0}}(X ; Y \mid \alpha=u)\right) d F_{\alpha}(u)=0,
$$

$\forall x \in \mathbb{R}$, and hence

$$
\begin{aligned}
& \int_{0^{+}}^{u_{0}}\left(-\int_{-\infty}^{\infty} P_{N}(y-u x) \log f_{Y \mid \alpha}(y \mid u) d y-D\right. \\
& \left.\quad-I_{F_{0}}(X ; Y \mid \alpha=u)\right) d F_{\alpha}(u)=0, \quad \forall x \in \mathbb{R} .
\end{aligned}
$$

We note that the exclusion of 0 from the bound of the integration in (43) does not affect the integral since the mutual infor- 
mation is zero if $u=0$. We now use the approach in $[5,13]$ Let us define $L(u)=I_{F_{0}}(X ; Y \mid \alpha=u)+\frac{1}{2} \log \left(2 \pi \sigma^{2}\right)$ and $\rho(y, u) \triangleq \log f_{Y \mid \alpha}(y \mid u)+L(u)$. Also define the sets

$$
\Omega_{u}^{+}=\{y: \rho(y, u) \geq 0\} \text {, and } \Omega_{u}^{-}=\{y: \rho(y, u)<0\} \text {. }
$$

We can then write,

$$
\begin{aligned}
\int_{0^{+}}^{u_{0}}\left[\int_{\Omega_{u}^{+}}\right. & P_{N}(y-u x) \rho(y, u) d y \\
& \left.\quad+\int_{\Omega_{u}^{-}} P_{N}(y-u x) \rho(y, u) d y\right] d F_{\alpha}(u)=0 .
\end{aligned}
$$

For the set $\Omega_{u}^{+}$, we have $\rho(y, u) \leq \log (\Gamma(y, u))+L(u) \leq$ $\log \left(k_{3}\right)+L(u)$. We define $l$ as

$$
l=\max _{u \in\left[0, u_{0}\right]} 2 u A+\sqrt{\frac{\log \left(k_{3}\right)+L(u)}{k_{4} \log (e)}} .
$$

Clearly, $\Omega_{u}^{+} \subseteq[-l, l]$. Therefore,

$$
\begin{aligned}
\int_{\Omega_{u}^{+}} P_{N}(y-u x) \rho(y, u) d y & \\
& \leq \int_{-l}^{l} P_{N}(y-u x) \rho(y, u) d y \\
& \leq\left(\log \left(k_{3}(u)\right)+L(u)\right) \int_{-l}^{l} Q(y-u x) d y
\end{aligned}
$$

which can be made arbitrarily small by choosing large values for $x$.

On the other hand for $x>l+A$,

$$
\begin{aligned}
\int_{\Omega_{u}^{-}} P_{N} & (y-u x) \rho(y, u) d y \\
& \stackrel{(a)}{\leq} \int_{l}^{\infty} P_{N}(y-u x) \rho(y, u) d y \\
& \leq \int_{l}^{\infty} P_{N}(y-u x)[\log (\Gamma(y, u))+L(u)] d y \\
& \stackrel{(b)}{<} \int_{x-A}^{x+A} q(A, u)[\log (\Gamma(x-A, u))+L(u)] d y \\
& =2 A q(A, u)[\log (\Gamma(x-A, u))+L(u)]<0
\end{aligned}
$$

where $(a)$ follows since $[l, \infty) \subset \Omega_{u}^{-}$while the integrand is negative, and $(b)$ follows since $q(A, u) \leq P_{N}(y-u x)$ and it is nonzero on its support by definition in (6), also the function $\log (\Gamma(y, u))+L(u)$ is monotonically decreasing in $y$ for $y>l$.

From (48) and (49), one can argue that $\forall u \in\left[0, u_{0}\right]$, there exists an $x \in \mathbb{R}$ such that the integration in (44) is strictly less than zero which contradicts with the optimality condition in (46), hence the set $E_{0}$ cannot have infinite number of mass points concluding the proof of the desired result.

Finally, we note that the channel capacity can be computed by finding the optimal input distribution and then evaluating the mutual information corresponding to this distribution. As we have shown, the capacity optimization problem is convex since the space of input distribution functions is convex and the mutual information is strictly concave. We also have shown that the capacity is achieved by a discrete distribution with a finite number of mass points. Thus, the problem of finding the optimal input distribution boils down to a finitedimensional convex optimization problem that aims to find the location of mass points and the associated probabilities corresponding to this distribution. To do this, an efficient numerical optimization algorithm can be developed which iterates over the number of mass points and its associated probabilities until the optimality conditions are satisfied and hence the optimal input distribution is found (similar to the approach in [1]).

\section{CONCLUSIONS}

We have studied the capacity of fading channels where the channel gain is only available at the receiver and the input is amplitude limited. We have shown that if the fading gain follows an arbitrary distribution with a finite support, the channel channel capacity is achieved by a unique optimal distribution and this distribution is discrete with a finite number of mass points.

\section{REFERENCES}

[1] J. G. Smith, "On the information capacity of peak and average power constrained Gaussian channels," Ph.D. dissertation, Department of Electrical Engineering, University of California, Berkeley, California, 1969.

[2] I. C. Abou-Faycal, M. D. Trott, and S. Shamai, "The capacity of discrete-time memoryless Rayleigh-fading channels," IEEE Transactions on Information Theory, vol. 47, no. 4, pp. 1290-1301, May 2001.

[3] M. C. Gursoy, H. V. Poor, and S. Verdú, "The noncoherent Rician fading channel-part I: structure of the capacity-achieving input," IEEE Transactions on Wireless Communications, vol. 4, no. 5, pp. 2193-2206, Sept. 2005.

[4] T. H. Chan, S. Hranilovic, and F. R. Kschischang, "Capacity-achieving probability measure for conditionally Gaussian channels with bounded inputs," IEEE Transactions on Information Theory, vol. 51, no. 6, pp. 2073-2088, May 2005.

[5] B. Mamandipoor, K. Moshksar, and A. K. Khandani, "On the sumcapacity of Gaussian MAC with peak constraint," in IEEE International Symposium on Information Theory Proceedings, July 2012, pp. 26-30.

[6] A. ElMoslimany, "A new communication scheme implying amplitude limited inputs and signal dependent noise: system design, information theoretic analysis and channel coding," Ph.D. dissertation, Department of Electrical, Computer and Energy Engineering, Arizona State University, Tempe, Arizona, 2015

[7] R. M. Gray, Entropy and Information Theory. Springer, 1990.

[8] M. S. Pinsker, Information and Information Stability of Random Variables and Processes. Izv. Akad. Nauk, 1960.

[9] J. G. Smith, "The information capacity of amplitude and varianceconstrained scalar Gaussian channels," Information and Control, vol. 18, no. 3, pp. 203-219, 1971.

[10] S. Boyd and L. Vandenberghe, Convex Optimization. Cambridge University Press, 2009.

[11] M. Fozunbal, S. W. McLaughlin, and R. W. Schafer, "Capacity analysis for continuous-alphabet channels with side information, part i: A general framework," IEEE Transactions on Information Theory, vol. 51, no. 9, pp. 3075-3085, 2005.

[12] J. W. Brown, R. V. Churchill, and M. Lapidus, Complex Variables and Applications. McGraw-Hill New York, 1996, vol. 7.

[13] A. Tchamkerten, "On the discreteness of capacity-achieving distributions," IEEE Transactions on Information Theory, vol. 50, no. 11, pp. 2773-2778, October 2004 